\title{
SOFT REAL TIME AUCTION SCHEME FOR TASK ALLOCATION IN WIRELESS SENSOR NETWORKS
}

\author{
Gangadharaiah S ${ }^{1}$, Umashankar M Hallur², Shanoor S Jamadar ${ }^{3}$ \\ ${ }^{1}$ Assistant Professor, Department of Information Science \& Engineering, Acharya Institute of Technology, Bangalore, \\ Karnataka, India \\ ${ }^{2}$ PG Student, Department of Information Science \& Engineering, Acharya Institute of Technology, Bangalore, Karnataka, \\ India \\ ${ }^{3}$ PG Student, Department of Information Science \& Engineering, Acharya Institute of Technology, Bangalore, Karnataka, \\ India
}

\begin{abstract}
In resource constrained wireless sensor networks, one of the fundamental challenges is to achieve a fair energy balance among nodes to maximize the overall network lifetime. Auction based schemes are one of the best method to maximize the network Lifetime. Existing Real Time Effective Auction (RTE-A), task allocation algorithm based on Auction method is not efficient in terms of evaluating the best bid value. As it solely based on real time auction method where successor of the current task may not be the best because, in the RTE-A the auction process is stopped as soon as auctioneer get bid value higher than threshold value. Real Time Effective Auction (RTE-A) algorithm is too stringent with respect to time constraints. But many applications may require resource allocation process which is flexible w.r.t time constraints. In our proposed algorithm a soft real time auction approach is used. Where winner of the auction process is not decided as soon as auctioneers get the bid value higher than threshold value But Auctioneer will have a particular time slot, which is based on application requirement. The auctioneer will decide winner at the end of the specific time slot based on maximum bid value. . In our proposed algorithm (SRT-A), we also improve real time task allocation approach by using wait time at the bidder side. The wait time method, tries to get the best bid value by allowing the node with highest bid value to bid earlier than other nodes. Proposed algorithm gives much better bid value in less time in real time application compare to RTE-A algorithm.
\end{abstract}

Keywords: Wireless Sensor Networks, Task Allocation, Auction Method, and Real Time Allocation

\section{INTRODUCTION}

Wireless Sensor Network (WSN) consists of hundreds or thousands of self-organizing, low-power, low cost wireless nodes and is used in a variety of applications such as military sensing and tracking, environmental monitoring, disaster management, etc

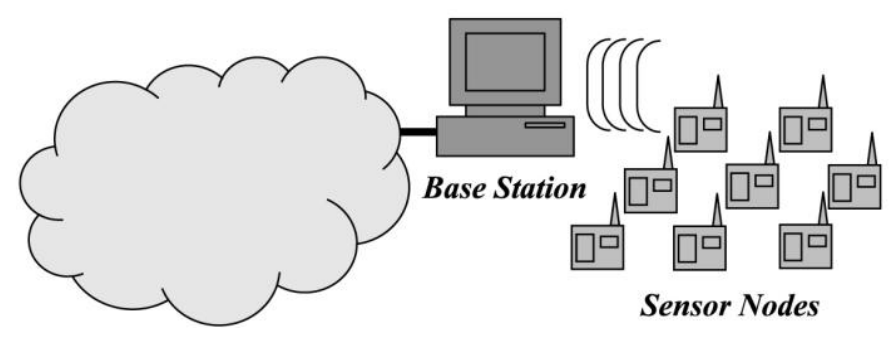

Fig -1: Scenario of Wireless Sensor Networks

Above Fig.1 shows the simple scenario of WSN [1], where wireless sensors collect data from the place where they are deployed and this gathered data is transferred to the sink via base station with the wireless communication. The sensors are embedded devices that are integrated with a physical environment and capable of acquiring signals, communicating and performing simple computational tasks.

While this new class of networks has the potential to enable wide range of applications, it also poses serious challenges like frequent network topology change, limited computational, memory and power supply. Wireless sensor networks are data centric. They are designed for specific application, compare to traditional network. To accommodate different type of applications, the design of WSN should be flexible in different level like hardware architecture, operating system, communication modules, routing algorithms, data fusion and aggregating methods, power management etc [2]. WSN provide smart method of computation. The data gathered from different sensors can be utilized to derive more meaningful context. But it also possesses many challenges like node should be cost effective, energy efficient, smaller size, stable and standard middleware. The network developed from this sensor should be self-configuring and fault tolerant, application specific and data centric. 
On the basis of information measured or carried the applications of WSN are categorized. Applications mainly concentrate on protocols and transmission techniques where as wireless channels constraints to communication capabilities and performance [3].

\section{LITERATURE SURVEY}

In this section, we discuss about related literature in various task allocation method in WSNs, different types of auction methods, resource allocation using auction method to improve the task allocation process.

\subsection{Task Allocation in WSNs}

As there are many issues present in the WSNs, the process of task allocation is very necessary in order to maintain the failure free operation of network. Some methods of task allocation are discussed below.

The work done in paper [4], discusses the Multi Attribute Utility Theory (MAUT). This paper tries to optimize the process of task allocation by defining energy consumption based utility function which helps in calculating the bid values in a more efficient manner. According to MAUT, the overall evaluation $\mathrm{v}(\mathrm{x})$ of an object $\mathrm{x}$ is defined as a weighted addition of its evaluation with respect to its relevant value dimensions the energy utility function.

The Work done in paper [5], aims for achieving a fair energy balance among sensor nodes with the help of task allocation, a market-based architecture called as the auction process. Here the nodes are modelled as sellers communicating a deployment price for problem, Centralized and distributed message exchange mechanisms between the nodes (seller) and task allocator (consumer) are proposed to determine the winner among the seller with the goal of reducing overhead and energy consumption. In Centralized message exchange method the consumer broadcast a message containing the (Task, Task size, Task Deadline) to all nodes each node calculate its cost (bid) and sends their bid to the consumer (bidder). The consumer selects minimum bid after evaluating the received bids from all the nodes, the major drawback of this approach is overhead and Collisions during message exchange is quite high. To overcome the disadvantage of centralized message exchange method Distributed message exchange scheme is used. In determination instead of accomplishing the current task immediately the nodes sets a waiting time and goes to a LISTEN mode. When the waiting time is completed the node would then send its cost to the consumer then the node with the lowest price will broadcast its bid first and be selected. After the winner is selected the remaining nodes which are in a LISTEN node will leave the competition.

The work done in paper [6], aims at achieving an efficient global behaviour for high performance, QOS and long network lifetime, allocate limited energy and radio bandwidth. An Autonomous Market-Based Approach is used for Resource Allocation in Cluster-Based Sensor Network. Resource allocation is calculated at each node at each round, winner has to be decided from resource bids during each round of scheduling which is very computation intensive, two schemas namely DRA (distributed resource allocation) and HRA (Hierarchical Resource Allocation) are used to improve global behaviour on network platform. In HRA schema sensor network is organized by cluster, communication backbone tree is formed by cluster head and some relay nodes. Each cluster heads has local centralized agent that can allocate the resource optimally in the cluster, each other node has a decentralized agent that decides the actions and resource that the node takes. At the base station there is a GPC (Global Price Controller) which provides client's control information such as resource price, this approach is not suitable for multi-hop, the main advantage is this approach is faster and accurate in tracking and avoids overlapping.

The work done in paper [7], proposes an auction based task allocation for power aware intrusion detection it defines the ATLAS algorithm [7], here calculation of PLANE metric of the nodes in the network and computing the threshold value based on the PLANE metric is done, here message exchange mechanism between nodes is an important energy consumption criteria. The types of messages used are (1) Request message: The message between coordinator and other nodes in the network. (2)Auction message: The message between coordinator and the set of monitoring nodes.

By varying the threshold value for better performance reduces communication cost and also regular updating of PLANE metric to maintain energy balance.

\subsection{Auction}

Auction [8] is a process of buying and selling goods or services by offering them up for bid, taking bids, and then selling the item to the highest bidder. The main reason is that auctions involve bidders competing simultaneously, and this benefits sellers as well as buyers. The different methods of auctioning are (1) First-price sealed-bid Auction: Here bidders place their bid in a sealed envelope. The auctioneer opens the bid and selects the highest who ends up paying exact amount as the highest bid. (2) Second-price sealed-bid Auction: Here bidders place their bid in a sealed envelope, The auctioneer opens the bid and selects the highest who ends up paying exact amount as the second highest bid.(3) Open ascending-bid Auctions (English auctions): Here the price is steadily raised by the auctioneer with bidders dropping out once the price becomes too high. The only bidder who remains at the end wins by paying the current price. (4) Open descending-bid Auctions (Dutch auctions): Here the bid starts with a high price high to deter all bidders it is progressively lowered. He or she wins the auction and pays the price at which they 
bid.(5)Sealed first-price auction, (FPSB): In this type of auction all bidders simultaneously submit sealed bids so that no bidder knows the bid of any other participant. The highest bidder pays the price they submitted.

The advantages of auction are (1) Competitive bidding often exceeds the expected value and returns the highest net value on a Seller's assets. (2) Seller knows exactly when the merchandise will be sold (time-defined sale). (3) An auction removes the seller from the "price negotiation" process. (4) An auction creates a buying frenzy and stimulates competition among buyers. (5) Auctions provide more efficient means to purchase merchandise.

\subsection{Auction in Resources Allocation}

The advantages of Resource Allocation by Auction method is (1) Auction reduces the overall communication cost (2) Auction creates competition among Bidders. (3) Auction helps in finding the more suitable successor. (4) Auction helps in maintaining energy values (5) Auction increases the value of resource.

\section{PROBLEM DEFINITION}

General Task allocation algorithm can't be applied to directly to sensor network due to its resource constraint such as limited computation capability, limited battery life, and limited communication bandwidth. Work done in [9] Real time Task allocation algorithm [RTA] based on Auction method is not efficient in terms of evaluating the best bid value. As it solely based on real time auction method where successor of the current task may not be the best because, in the existing algorithm the auction process is stopped as soon as auctioneer get bid value higher than threshold value(expected minimum Bid value). And also existing algorithm never tries to maintain energy balance in the network as it allows each and every node in the network to take part in the auction process without taking in to consideration about the maximum amount of energy left in every node.

\section{PROPOSED WORK}

Our proposed algorithm is based on soft real time auction approach. In this algorithm, the winner of the auction process is not decided as soon as auctioneers get the bid value higher than threshold value. But Auctioneer will have a particular time slot, which is based on application requirement. The auctioneer will decide winner at the end of the specific time slot based on maximum bid value. This algorithm gives a fair chance to all nodes which have sufficient energy to participate in the auction, there by maintaining the energy balance in the network. In our algorithm we also implement the wait time approach where we introduce the wait time and allow the bidders to bid based on their wait time, here with the help of wait we can make the node with highest bid value to bid first as the wait time is derived based on the bid values of each node.

\section{DESIGN}

In this section, we provide the description of the proposed algorithm i.e. Soft Real-Time Auction (SRT-A).

The less energy node (auctioneer) will call for an auction process to allocate its task to other nodes in the network (bidders).On receiving the auction message the bidders will bid their values then the auctioneer will compare the bid value and chose the maximum bid at the end of the each slot. In second case we decide based on Wait time. Wait time derived for Energy utility value. This algorithm gives a fair chance to all nodes to participate in the auction as the winner is chosen at the end of the time slot and also the algorithm gives more preference to the nodes with max bid to facilitate an efficient task allocation process. The algorithm is divided into two sections the Bidder side and the Auctioneer side. The working of Auctioneer side is described below.

\subsection{Soft Real Time Auction (SRT-A) Algorithm}

In SRT-A algorithm the less energy node (auctioneer) will call for an auction process to allocate its task to other nodes in the network (bidders).On receiving the auction message the bidders will bid their values then the auctioneer will compare the bid value and chose the maximum bid at the end of the each slot. In second case we decide based on Wait time. Wait time derived for Energy utility value. This algorithm gives a fair chance to all nodes to participate in the auction as the winner is chosen at the end of the time slot and also the algorithm gives more preference to the nodes with max bid to facilitate an efficient task allocation process. The algorithm is divided into two sections the Bidder side and the Auctioneer side. The working of Auctioneer side is described below.

\subsubsection{Auctioneer Algorithm for SRT-A}

The steps in the Soft Real-Time Auction (SRT-A) auctioneer algorithm are illustrate as below Begin

1) The node with less energy i.e. the auctioneer which cannot accomplish its current task will broadcast the task to the other nodes in the network i.e. the bidders, and goes to wait mode until the auction ends.

2) When the bid time ends the simulation will be stopped and the bid value from bidders will be saved.

3) The auctioneer will compare the bid value with the threshold value and checks the following condition. If (bidvalue>maxvalue) It waits for other bidders to, to bid their value and again checks the following condition.

If (new bidvalue>threshold)

Maxvalue=new bidvalue; 
Maxvalue=bidvalue;

4) In SRT-A, the auctioneer will wait until the end of time slot and decide the winner. The size of this time slot can be tuned as per application requirement.

End

5) The simulation is stopped.

\subsubsection{Bidder side Algorithm for SRT-A}

The steps in the Soft Real-Time Auction (SRT-A) Bidder side algorithm are illustrate as below

Begin

1) Bidders receive the maxvalue and values of Vload and Wload from the auctioneer.

2) Upon receiving the maxvalue the bidders will calculate their individual energy utility value i.e. the bid value using the energy consumption based utility function as shown below $\mathrm{U}(\mathrm{O})=$ Vload $*$ Wload + Venergy* Wenergy

3) Each node will wait for random amount of time

4) Send the bid value to Auctioneer

End

5) Stop the thread.

\subsection{Wait Time Based Real Time Auction (WBRT-A)}

\section{Algorithm}

In this proposed algorithm, Wait Time based Real-Time Auction (WBRT-A) the node with more energy will bid at first. In bidder side, nodes are asked to bid based on energy utility value. Once the energy utility value is calculated, wait time is derived based on this value.

\subsubsection{Auctioneer Algorithm for WBRT-A}

The steps in the Wait Time based Real-Time Auction (WBRTA) algorithm are illustrate as below

1) Task publishing model is broadcasted by the auctioneer. It will consist of a maxvalue and required values for load and energy.

2) On receiving the task publishing model the bidders will calculate their bids using the energy consumption based utility function and send their bids to the auctioneer. The bid is calculated as shown below.

$\mathrm{U}(\mathrm{O})=$ Vload $*$ Wload + Venergy* Wenergy.

3) Upon receiving the bids the auctioneer will evaluate the bids based on the following cases.

Case 1: Real-Time Effective Auction(RTE-A)

If(bidvalue >maxvalue)

BidMax=bidvalue;

The auction is stopped and the current max bidder is chosen as the winner of the auction process.

Case 2: Real-Time Effective-NonFailure Auction

(RTENF-A)
BidMax $=\max ($ bidvalue $)$;

Here the node which has max bid value among all the nodes is chosen as the winner of auction process.

4) Once time deadline is over, auction process is terminated.

End

\subsubsection{Bidder Algorithm for WBRT-A}

The steps in the Wait Time based Real-Time Auction (WBRT-

A) Bidder side algorithm are illustrate as below.

Begin

1) Bidders receive the maxvalue and values of Vload and Wload from the auctioneer.

2) Upon receiving the maxvalue the bidders will calculate their individual energy utility value i.e. the bid value using the energy consumption based utility function as shown below

$\mathrm{U}(\mathrm{O})=$ Vload $*$ Wload + Venergy* Wenergy

3) Each node will wait for time calculated based on energy utility value.

4) Send the bid value to Auctioneer

End

5) Stop the thread.

\section{SIMULATION}

With the proposed algorithm, we conducted simulation by using Java Threads. Each node is assumed to be thread, for experimental purpose we have conducted the simulation for 5 to 30 nodes. We have considered parameters like (1) Best bid value (2) Time taken to get best bid value and (3) Number of possible best bid values for performance evaluation.

The below screenshots Fig.2 and Fig.3 shows the output from SRT-A and WBRT-A algorithms.

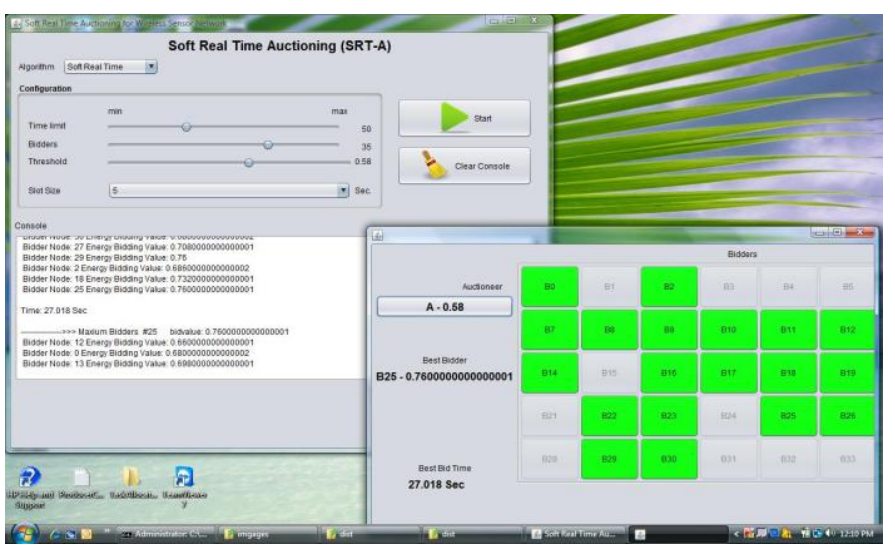

Fig -2: Screen shot of SRT-A scheme 


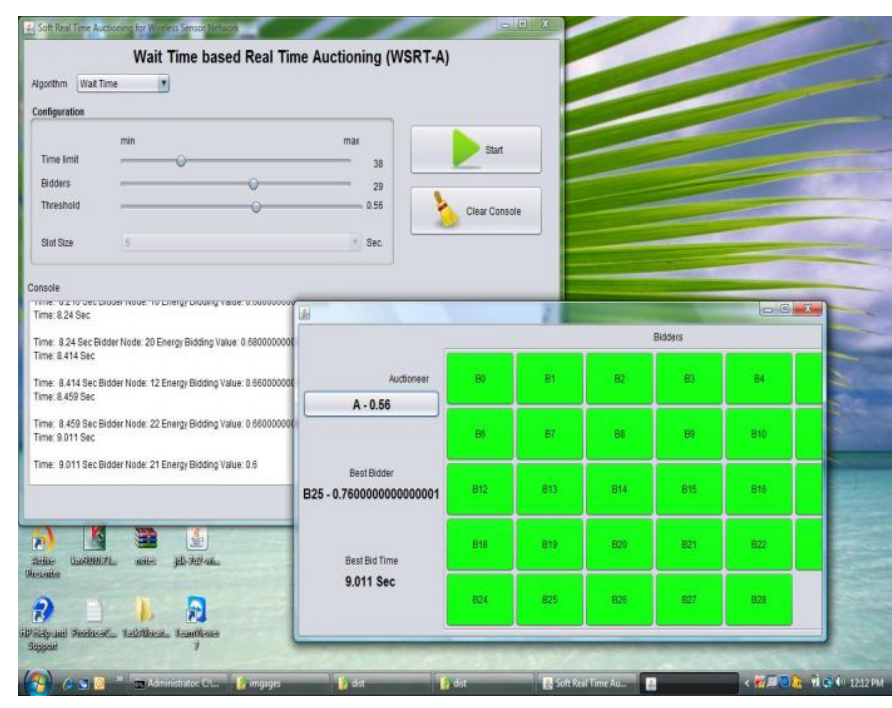

Fig -3: Screen shot of WBRT-A scheme

\section{RESULTS AND DISCUSSIONS}

\subsection{Analysis of bid time performance in RTE, SRT,}

\section{WBRT}

In this test analysis, we compare the bidding time performance of RTE-a, SRT-A, and WBRT-A by varying the threshold value

\subsubsection{Analysis of Bid Time Performance for} Threshold Value 0.60

Time Taken to generate Best bid Value

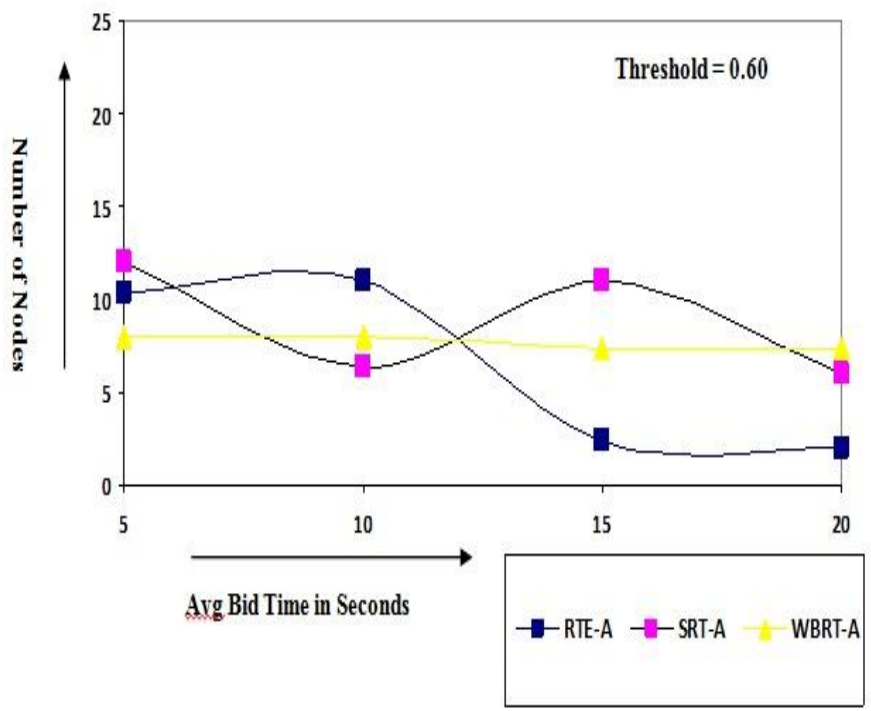

Fig -4: Time taken to generate bid value with thrld=0.60

\subsubsection{Analysis of Bid Time Performance for} Threshold Value 0.70

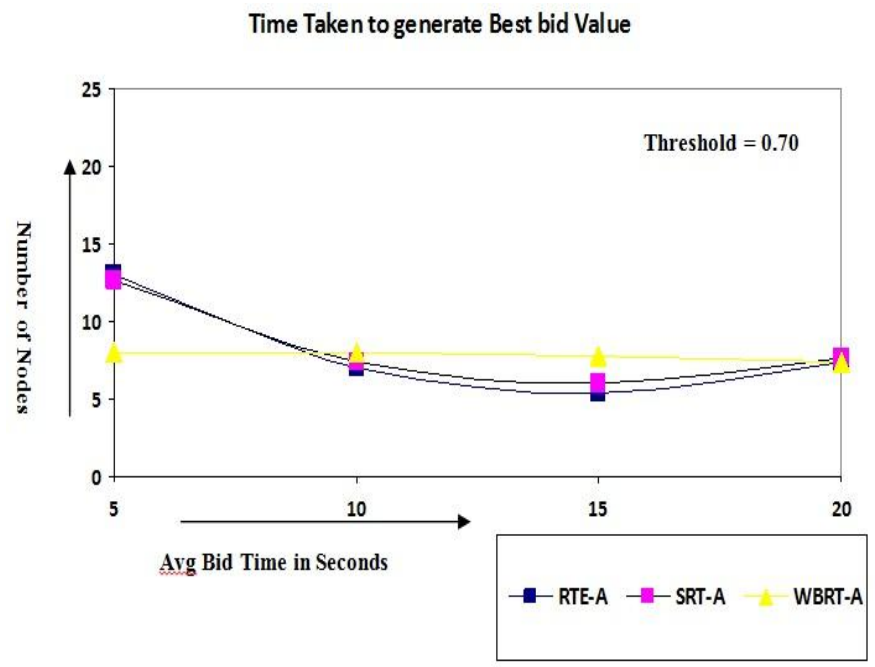

Fig -5: Time taken to generate bid value with thrld $=0.70$

\subsection{Analysis of Bid Time Performance in RTE, SRT}

In this test analysis, we compare the number of bids generated in RTE-a, SRT-A, and WBRT-A by varying the threshold value.

\subsubsection{Analysis of Bid Generation Performance for} Threshold Value 0.70

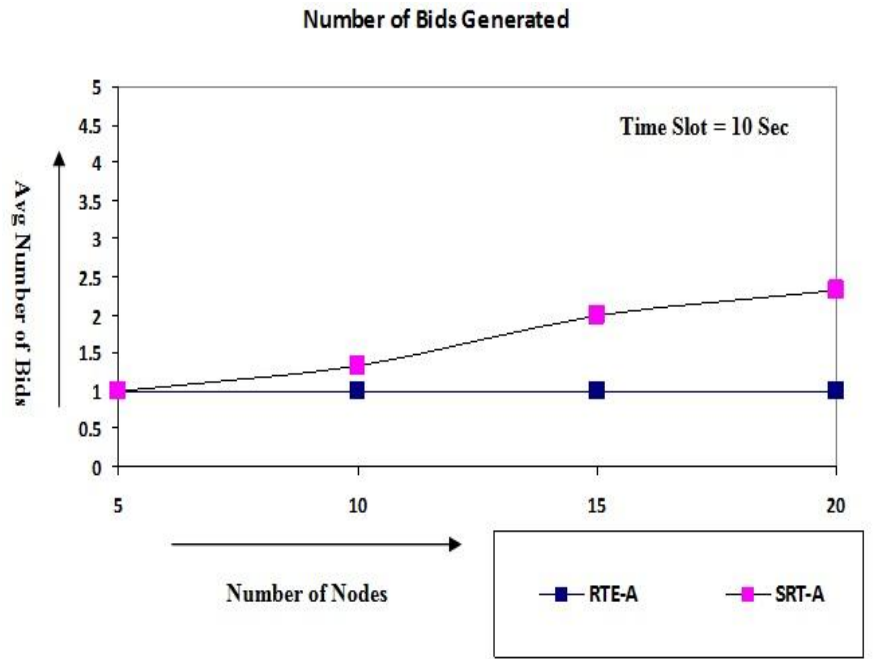

Fig -6: Number of bids generated with thrld=0.70 


\subsubsection{Analysis of Bid Generation Performance for}

\section{Threshold Value 0.60}

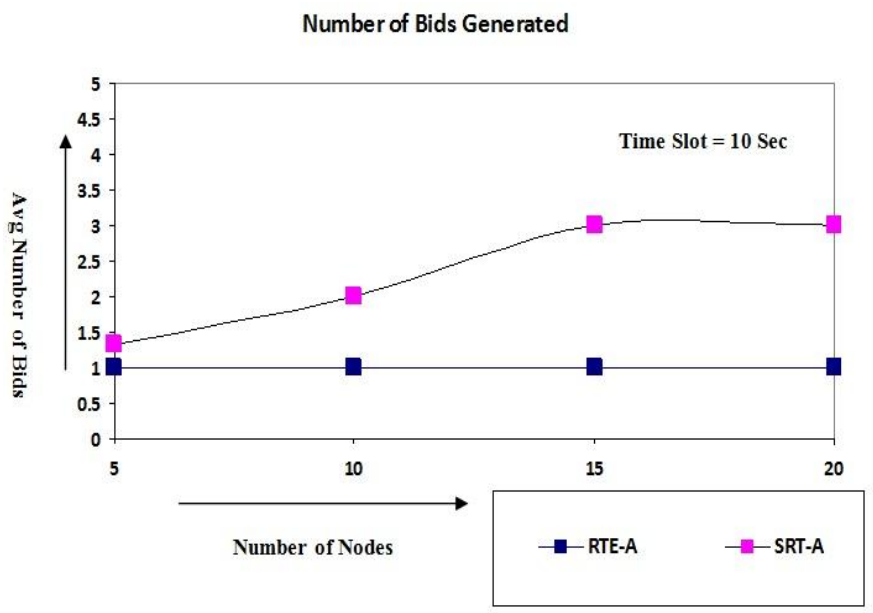

Fig -7: Number of bids generated with thrld $=0.60$

\subsection{Analysis Performance SRT-A by varying the}

\section{Time Slot}

In this test analysis, we compare performance of SRT-A by varying the time slot.

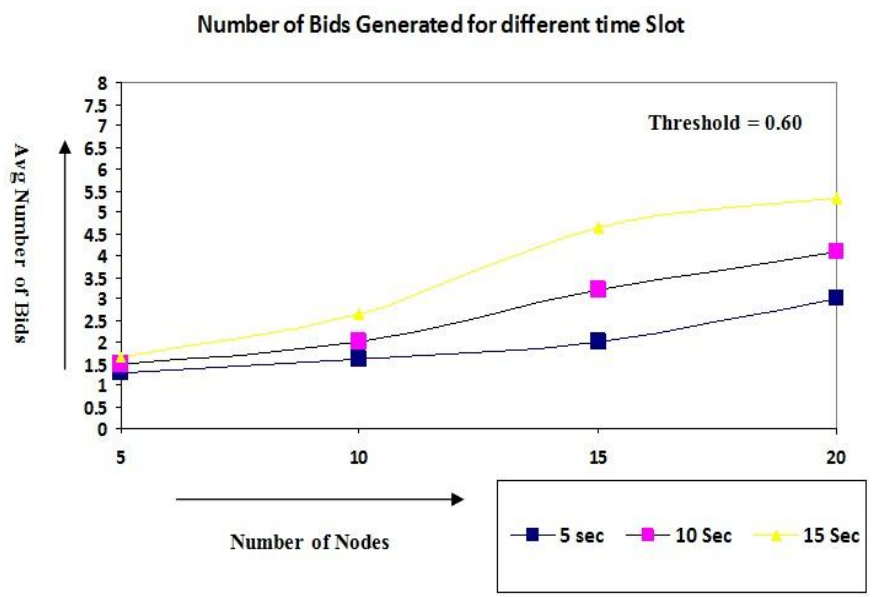

Fig -8: Number of bids generated for different time slot

\section{CONCLUSIONS AND FUTURE WORK}

Our proposed algorithm is based on soft real time auction approach. In this algorithm, the winner of the auction process is not decided as soon as auctioneers get the bid value higher than threshold value. But Auctioneer will have a particular time slot, which is based on application requirement. The auctioneer will decide winner at the end of the specified time slot based on maximum bid value. This algorithm gives a fair chance to all nodes which have sufficient energy to participate in the auction, there by maintaining the energy balance in the network. In our algorithm, we improve real time approach by using wait time at the bidder side. The wait time method, tries to get the best bid value by allowing the node with highest bid value to bid earlier than other nodes. It gives much better bid value in less time in real time application.

The result shows that the number of bid generated per auction process will increase with increasing time slot. We also improve real time task allocation approach by using wait time at the bidder side. The wait time method, tries to get the best bid value by allowing the node with highest bid value to bid earlier than other nodes. It gives much better bid value in less time in real time application.

In future, we will be looking forward to port the proposed algorithm to more realistic simulation environment to study and to improvise the proposed algorithm.

\section{REFERENCES}

[1]. Rodrigo Roman, Javier Lopez, (2009) "Integrating wireless sensor networks and the internet: a security analysis", Internet Research, Vol. 19 Iss: 2, pp.246 - 259

[2].http://www.academia.edu/2468712/Wireless_Condition_M onitoring_and_Embedded_Novelty_Detection

[3]. Wei-Lun Chang, Using Multi-attribute Utility Theory to Rank and Select Co-branding Partners, In: Proceedings of Intelligent Systems Design and Applications, 2008. ISDA '08. Eighth International Conference on, 2008(1), pp,195-200.

[4]. A price-based adaptive task allocation for Wireless Sensor Network Edalat, N.; Wendong Xiao; Chen-Khong Tham; Keikha, E.; Lee-Ling Ong Mobile Adhoc and Sensor Systems, 2009. MASS '09. IEEE 6th International Conference on Digital Object Identifier: 10.1109/MOBHOC.2009.5337039 Publication Year: 2009

[5]. Wei-Lun Chang, Using Multi-attribute Utility Theory to Rank and Select Co-branding Partners, In: Proceedings of Intelligent Systems Design and Applications, 2008. ISDA '08. Eighth International Conference on, 2008(1), pp,195-200.

[6]. T. Srinivasan, V. Vijaykumar, and R. Chandrasekar, An Auction based Task Allocation Scheme for Power-Aware Intrusion Detection in Wireless Ad-Hoc Networks, In: Proceedings of IFIP on Wireless and Optical Communication Networks, Bangalore, 2006, pp. 0-5

[7]. http://en.wikipedia.org/wiki/Auction

[8]. Lokesh. B. Bhajantri, Nalin. N, Gangadharaiah .S, "Context Aware Resource Allocation in Distributed Sensor Network", International Journal of Wireless \& Mobile Networks (IJWMN) Vol. 4, No. 2, April 2012 


\section{BIOGRAPHIES}

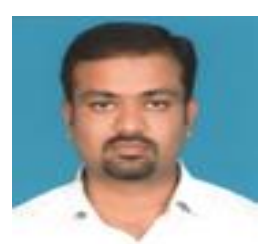

Gangadharaiah S. received the B.E. and M.Tech. Degree in Computer Science \& Engineering from Visvesvaraya Technological University, Karnataka in 2003 and 2011, respectively. $\mathrm{He}$ is an Assistant professor in the Department of Information Science \& Engineering, Acharya Institute of Technology, Bangalore. His research interests include wireless sensor network, Embedded Systems.

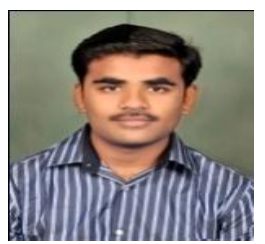

Umashankar Hallur, pursuing Master of Technology in Computer Network and Engineering, Department of Information Science and Engineering, Acharya Institute of Technology, Bangalore, Karnataka, India. His areas of interest are Networking and Wireless Sensor Networks.

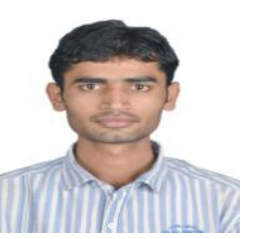

Shanoor S Jamadar, pursuing Master of Technology in Computer Network and Engineering, Department of Information Science and Engineering, Acharya Institute of Technology, Bangalore, Karnataka, India. His areas of interest are Networking and Wireless Sensor Networks 